\title{
Commissioning of the ATLAS Electron, Photon and Tau Trigger Selection
}

\author{
Clemencia Mora Herrera on behalf of the ATLAS Collaboration
}

\begin{abstract}
Since March 2010, the ATLAS experiment has been recording proton-proton collisions of the Large Hadron Collider (LHC) at a center of mass energy of $7 \mathrm{TeV}$. At low instantaneous luminosity, data were selected by the hardware based Level-1 trigger and processed by the software based High Level Trigger (HLT) without active rejection; as the luminosity increased, the HLT rejection has been gradually activated. Since then, electrons from $\mathrm{J} / \psi$, bottom, charm, $\mathrm{W}$ and $\mathrm{Z}$ decays, prompt photons and a first sample of tau hadronic decays from $W$ have been efficiently selected. This paper gives an overview of the implementation of the electron, photon and tau trigger selection algorithms and of the first experience running these triggers online. The performance of the three trigger levels is discussed and a set of comparisons of the online discriminating variables with offline reconstruction is shown, as well as the comparison of data with the Monte Carlo simulation on which the current selection was tuned.
\end{abstract}

\section{INTRODUCTION}

ATLAS is one of the two multipurpose experiments that operate at the Large Hadron Collider (LHC). At the center of the ATLAS detector, proton bunches collide at $7 \mathrm{TeV}$ center of mass energy. It is composed (from the centre of the detector outwards) of the inner detector trackers; a superconducting solenoid, generating a magnetic field of $2 \mathrm{~T}$; a Liquid Argonlead electromagnetic calorimeter; a hadron calorimeter; and a muon spectrometer system. At the LHC design luminosity of $10^{34} \mathrm{~cm}^{-2} \mathrm{~s}^{-1}$ the bunch crossing rate is around $40 \mathrm{MHz}$, whereas the data recording rate is limited to some $200 \mathrm{~Hz}$, constrained by technology and available resources. Thus only one out of $O\left(10^{5}\right)$ events will be recorded by ATLAS for analysis; the rest will be rejected by the trigger system. The ATLAS trigger system is divided into three levels: the hardware-based first level trigger, and the software based second-level and Event Filter triggers, jointly called the High Level Trigger (HLT). During the commissioning phase, data were actively selected by the L1 trigger and subsequently processed online by the HLT, which accepted all events, independent of the result of the selection. As luminosity increased and HLT algorithms were validated, thresholds were gradually adjusted, and HLT selection was fully activated when the instantaneous luminosity reached $\mathcal{L}=2 \times 10^{30} \mathrm{~cm}^{-2} \mathrm{~s}^{-1}$, in late July 2010. Fig.1 shows the increase in instantaneous luminosity as a function of the date for the whole 2010 datataking period, up to the beginning of November.

Ongoing analyses by the ATLAS collaboration are using the HLT triggers as part of their primary selections, for the full dataset of $45 p b^{-1}$.

C. Mora Herrera is with the University of Geneva, Quai Ernest-Ansermet 24, CH-1211 Genève, Switzerland; e-mail: Clemencia.Mora.Herrera@cern.ch

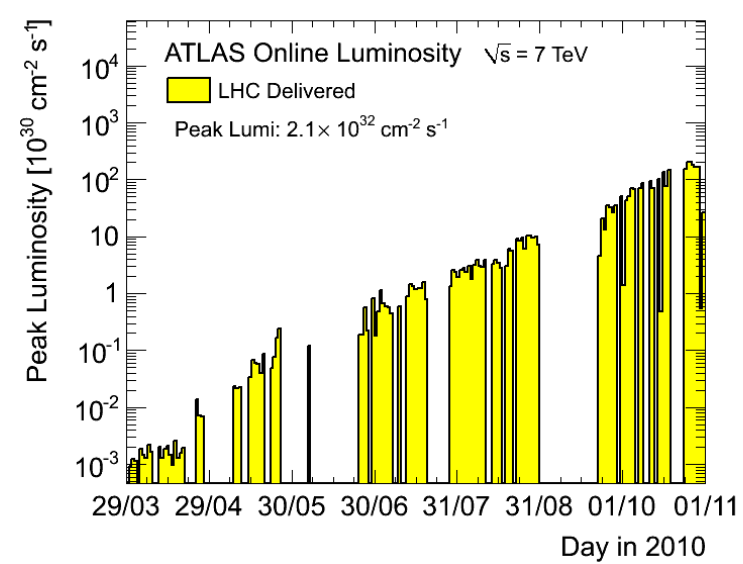

Figure 1. Peak instantaneous luminosity seen by the ATLAS detector, as a function of the date. The High Level Trigger rejection was activated in late July 2010.

In the following sections the ATLAS trigger selection for electrons, photons, and tau leptons will be described, and an overview of the performance of the first level and the HLT for these triggers will be shown.

\section{THE ATLAS DETECTOR}

The ATLAS detector consists of several concentric detectors. The innermost layer contains tracking detectors, made of silicon pixels, silicon microstrip and transition radiation detectors, surrounded by a solenoid magnet coil that gives a magnetic field of $2 T$ in the direction of the beam. The next layer going away of the interaction point contains the calorimeters, where electrons, photons and jets are stopped and their energies measured. They consist of the electromagnetic (EM) and hadronic (HAD) components. The EM calorimeter uses liquid Argon (LAr) as sampling material, while the HAD calorimeter uses scintillating tiles in the barrel region and LAr in the end-caps. The outermost layer is the muon spectrometer, comprised of muon chambers and a toroidal system of magnets, to measure the momentum of muons. The ATLAS detector effectively covers the angular region corresponding to $|\eta|<2.5$ for tracking and $|\eta|<$ 4.9 for calorimetry (with the electromagnetic calorimeter extending up to $|\eta|<3.2$ ). As the inner detector and the calorimeters are relevant to the triggers discussed in this paper, a more thorough description of these detector systems is given here. A more general description of the detector can be found elsewhere [1]. 
The inner tracking detector combines high resolution detectors at the inner radii with continuous tracking elements at the outer radii. From the inside to the outside it consists of a three-layer semiconductor pixel detector, a semiconductor strip detector (SCT) with 4 layers in the barrel region and 9 layers in each of the end-caps, both of which cover the region $|\eta|<$ 2.5; and a transition radiation tracker composed of straw-tube proportional chambers interleaved with a radiator material that covers the region $|\eta|<2.0$. The expected tracking resolution is $\sigma_{p_{T}} / p_{T}=0.05 \% p_{T} \oplus 1 \%$, where $p_{T}$ is the transverse momentum expressed in $\mathrm{GeV}$.

The electromagnetic calorimeter uses lead as absorber and has an accordion geometry that gives a very good spatial uniformity in the azimuthal angle, $\phi$, and is divided into three longitudinal layers. It is expected to achieve an energy resolution of $\sigma_{E} / E=10 \% / \sqrt{E} \oplus 0.7 \%$ where $E$ is the energy expressed in $\mathrm{GeV}$ [1]. The size of calorimeter cells varies with pseudo-rapidity and with the calorimeter layer. Layer 1 has a very fine granularity of $\Delta \eta \times \Delta \phi=0.003 \times 0.1$ in the barrel region and slightly coarser granularity up to $|\eta|<$ 2.4. Electromagnetic showers deposit most of their energy in layer 2 which has a granularity in $|\eta|<2.5$ of $\Delta \eta \times \Delta \phi=$ $0.025 \times 0.025$.

The hadron calorimeter covers the range $|\eta|<4.9$ exploiting different techniques. In the barrel region it is a sampling calorimeter using steel as the absorber material and scintillating tiles as active material. In the range $1.5<|\eta|<4.9$ Liquid Argon calorimetry takes over: the end-cap hadron calorimeter covers $|\eta|<3.2$ while the range $3.1<|\eta|<4.9$ is covered by the high-density forward calorimeter. The typical size of a readout cell in the hadron calorimeter is $\Delta \eta \times \Delta \phi=0.1 \times$ 0.1 in the Tile and hadronic end-cap calorimeter and $0.2 \times$ 0.2 in the forward calorimeter. The expected resolution for measuring jets is $\sigma_{E} / E=50 \% / \sqrt{E} \oplus 3 \%$ in $|\eta|<3.2$ and $\sigma_{E} / E=100 \% / \sqrt{E} \oplus 10 \%$ in $|\eta|<4.9$.

The Minimum Bias Trigger Scintillators (MBTS), used for selecting good collision events for some of the studies shown here, cover the region $2.09<|\eta|<3.84$ and are mounted at $|z|=3.6 \mathrm{~m}$ between the inner tracking detector and the electromagnetic end-cap calorimeter [2].

\section{The ATLAS Trigger Selection of Electrons, PHOTONS AND TAUS}

The ATLAS trigger system is designed to select interesting physics events from LHC collisions. In order to do this, it identifies high transverse-momentum particles as well as events with large missing or total transverse energy. The first level (L1) uses fast custom-built electronics and takes input from the muon system, the calorimeters and the minimum bias scintillators with coarse granularity. At the High Level Trigger stage the information from the tracking detectors is included as well. The Level 2 (L2) trigger uses fast algorithms to refine the selections, taking information from a small portion of the detector, called the Region of Interest (RoI), defined by the L1 trigger. The final level, called the Event Filter (EF), uses algorithms from offline reconstruction and can access the full event readout as well as data from the RoIs. The general description of the trigger system [3] and the performance of the muon selection [4] are discussed separately in these proceedings.

Electrons and photons leave most of their energy in the electromagnetic calorimeter, and electrons also have a track in the inner detector. Tau leptons decay in the beampipe and, depending on the decay mode, are either detected through their leptonic products or with specialized algorithms for the hadronic decay mode. A hadronically decaying tau lepton looks like a narrow jet in the calorimeters with a few tracks in the inner detector. These characteristics are exploited by the trigger in order to identify electrons, photons and hadronic decays of taus.

For the comparisons presented here, the Monte Carlo distributions are normalized to the number of events in the data sample

\section{A. Performance of the Level 1 Selection of Electrons, Photons and Taus}

For the L1 selection of electrons, photons and hadronic taus, the ATLAS calorimeter is segmented into electromagnetic and hadronic trigger towers (TT) which are sums of calorimeter cell signals with a granularity of $0.1 \times 0.1(\eta \times \phi)$. The e- $\gamma$ and $\tau$ candidates are found by a sliding window algorithm of $4 \times 4$ trigger towers. The cluster candidate must satisfy these conditions:

- The central $2 \times 2$ trigger tower sum must be a local $E_{T}$ (transverse energy) maximum.

- The cluster energy for e- $\gamma$ is the highest $2 \times 1$ or $1 \times 2$ sum of EM trigger towers, where the $\tau$ cluster energy is defined as the EM cluster energy plus the central $2 \times 2$ HAD trigger towers sum. This energy has to be above a configured threshold.

- If isolation is required, the total $E_{T}$ in the isolation region has to be less than a configured isolation threshold. Fig.2 shows diagrammatically the definition of the isolation regions and the sums used. Isolation is not currently being required at the Level 1 selection, but the capability is present.

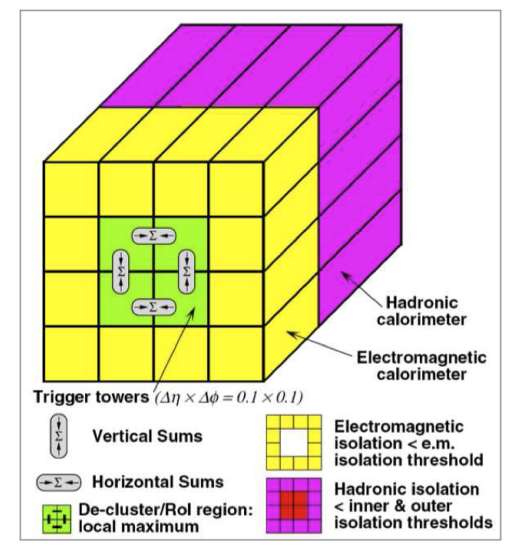

Figure 2. Diagram showing the basic objects used for Level 1 calorimeter triggers. The ATLAS $\mathrm{e} / \gamma$ and $\tau$ selections use the same hardware for this step. 
In order to understand the performance of the trigger, its efficiency in finding offline reconstructed candidates needs to be measured. The data have been selected with the Minimum Bias trigger in order to obtain an unbiased sample for estimating the L1 trigger efficiencies for tau and e- $\gamma$.

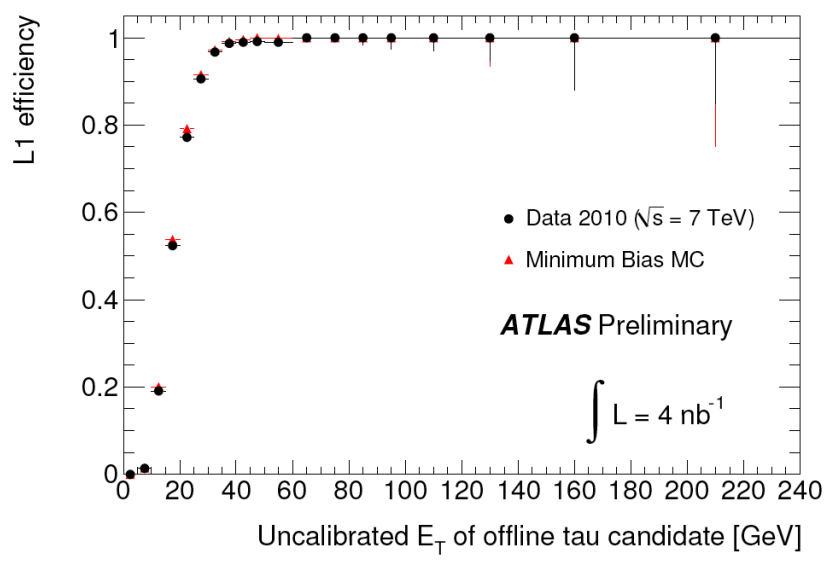

Figure 3. Efficiency for L1 tau trigger with a threshold of $5 \mathrm{GeV}$, as a function of the offline tau candidate uncalibrated $E_{T}$.

Fig. 3 shows the fraction of the offline tau candidates which match geometrically to a L1 tau trigger object with $E_{T}>5$ $\mathrm{GeV}$. Each offline tau candidate should have associated with it at least one charged track with $p_{T}>1 \mathrm{GeV}$ and a minimum of seven hits in the silicon detectors. Other clean up procedures have also been employed as described in [5]. This fraction is shown as a function of the uncalibrated $E_{T}$ of the offline tau candidate. Good agreement between the data and the MonteCarlo simulation is observed, especially at high transverse energies.

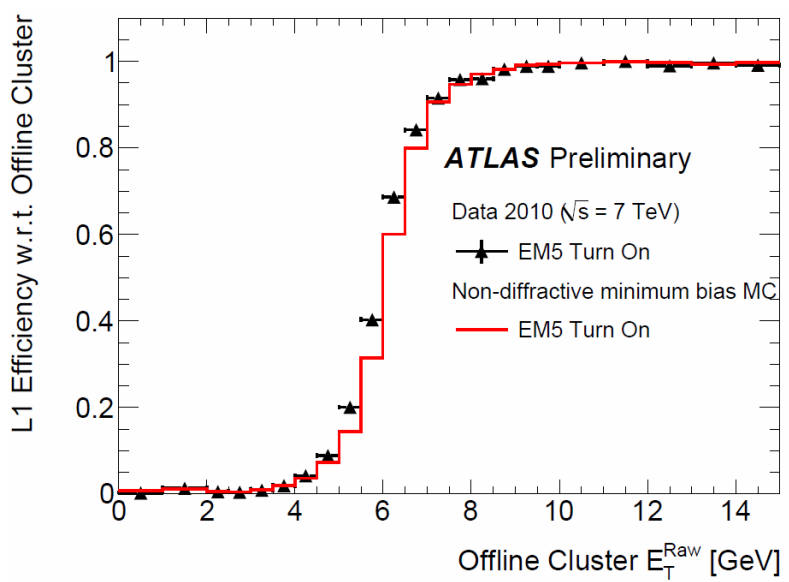

Figure 4. Efficiency of the L1 e- $\gamma$ trigger for a threshold of $5 \mathrm{GeV}$, as a function of the raw transverse energy of the offline EM cluster.

The L1 efficiency for the trigger selecting EM clusters above $5 \mathrm{GeV}$ as a function of the uncalibrated offline cluster $E_{T}$ is shown in Fig.4. The efficiency turn-on is shown for data (markers) and Monte Carlo simulation (lines). The efficiency turn-on in data occurs at slightly lower $E_{T}$ values than Monte Carlo and the shape is well modelled.

\section{B. Performance of the HLT Selection of Electrons and Photons}

The L2 photon and electron selection deploys a fast calorimeter reconstruction algorithm and for electrons also fast track reconstruction [6]. Identification of good electron and photon candidates over jet background are based on analyzing the cluster shower shapes, and for electrons also the tracking information and cluster-track matching quantities.

The EF uses the offline reconstruction algorithms adapted for online timing constraints, e.g. bremsstrahlung recovery is not applied. In a manner similar to L2, it utilizes the information from calorimeter shower shapes, and also tracking in the case of electrons, to select the good e- $\gamma$ candidates. One important feature of the HLT e- $\gamma$ triggers is the use of the same or a subset of the selection criteria used by the offline particle identification. This guarantees a minimal trigger bias in offline physics analyses [7].
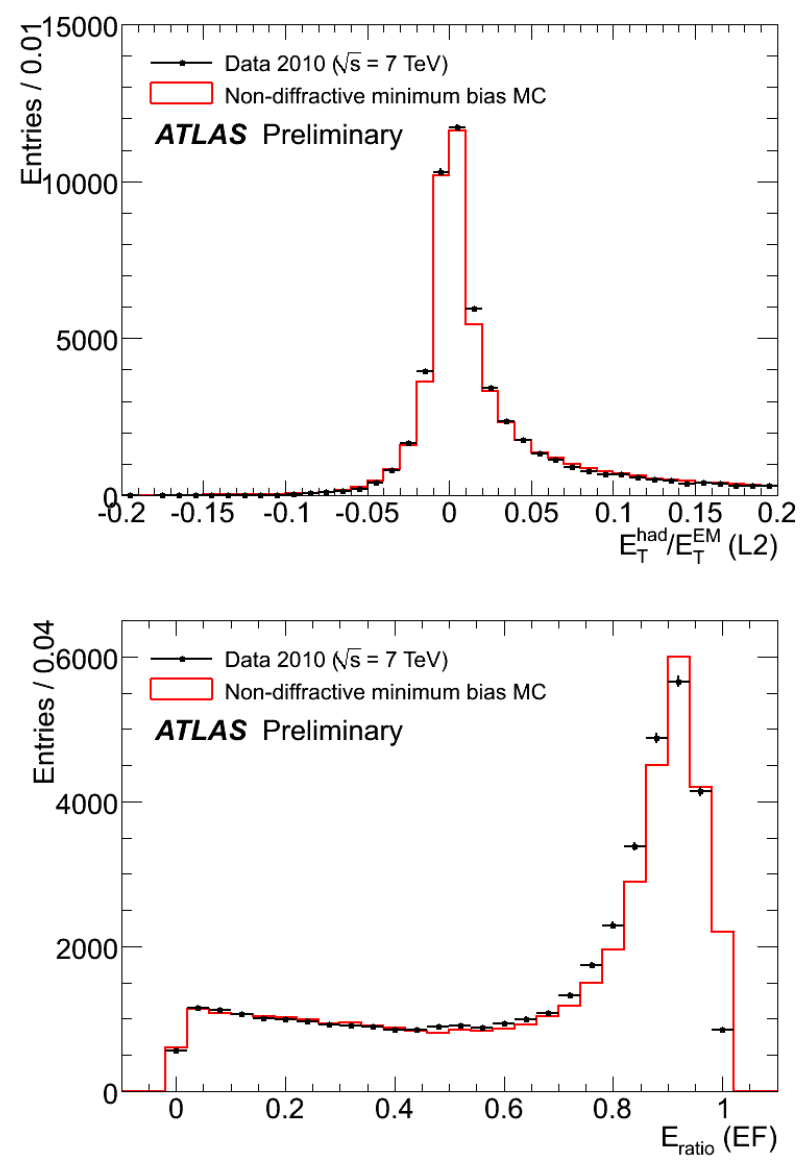

Figure 5. Comparison of the distribution of calorimeter variables used by the HLT to identify electrons and photons between Monte-Carlo and collisions data with $\sqrt{s}=7 \mathrm{TeV}$. The upper plot shows the ratio of the cluster energy detected in the hadronic calorimeter over the one in the electromagnetic calorimeter, the so-called hadronic leakage. The bottom plot shows the variable $E_{\text {ratio }}$, which is useful for rejecting $\pi^{0} \rightarrow \gamma \gamma$.

Fig. 5 shows a good agreement between measured and predicted distributions for two of the calorimeter discriminating 
variables; the ratio of the hadronic to the electromagnetic cluster energy calculated at Level 2 , which allows rejecting fakes depositing energy in the hadronic calorimeter; and the variable $E_{\text {ratio }}$ for $\mathrm{EF}$, defined for the first layer of the electromagnetic calorimeter as:

$$
E_{\text {ratio }}=\frac{E_{1}^{\max }-E_{2}^{\max }}{E_{1}^{\max }+E_{2}^{\max }}
$$

where $E_{1}^{\max }$ is the energy of the most energetic cell, and $E_{2}^{\max }$ is the energy of the second highest energetic cell isolated from the first peak. This variable helps in discriminating good electrons and photons from $\pi^{0}$ decaying to two photons.

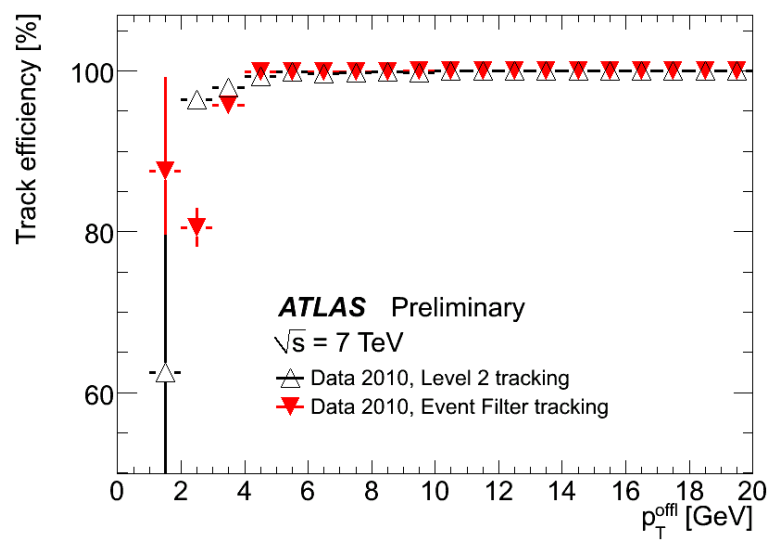

Figure 6. High Level Trigger tracking efficiency with respect to good offline electron candidates. The high efficiency shows that HLT tracking algorithms will find a track for nearly all electrons with $p_{T}>5 \mathrm{GeV}$.

The selection of electrons by the HLT also requires reconstruction of a track. As seen in Fig.6 electrons with $p_{T}>5 \mathrm{GeV}$ have $100 \%$ efficiency for having their tracks reconstructed by the HLT algorithms.

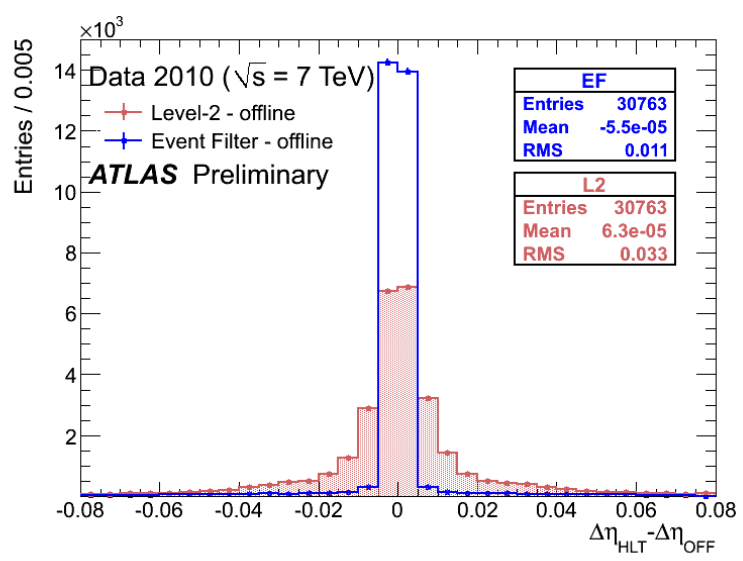

Figure 7. Resolution of $\Delta \eta$ for L2 in red (dot-filled) and EF in blue hollow line, for collisions data at $\sqrt{s}=7 \mathrm{TeV}$.

The electron's track is required to geometrically match its cluster position. The distance between the cluster and the track in $\eta, \Delta \eta$, is one parameter used to identify good electrons.
Fig. 7 shows the resolution of this variable for L2 and EF with respect to the $\Delta \eta$ found for offline electrons. As can be seen in the figure, this is better for the Event Filter identification, since the Level 2 has to use fast, less accurate algorithms.

\section{Performance of the HLT Selection of Taus}

The tau reconstruction at L2 starts from the RoI defined by L1, building a cluster centered at the L1 RoI position. Tracking and calorimeter information are used by the L 2 algorithms to apply selection criteria, where the narrowness of the jet and the low track multiplicity allows discriminating between tau candidates and multi-jet background.
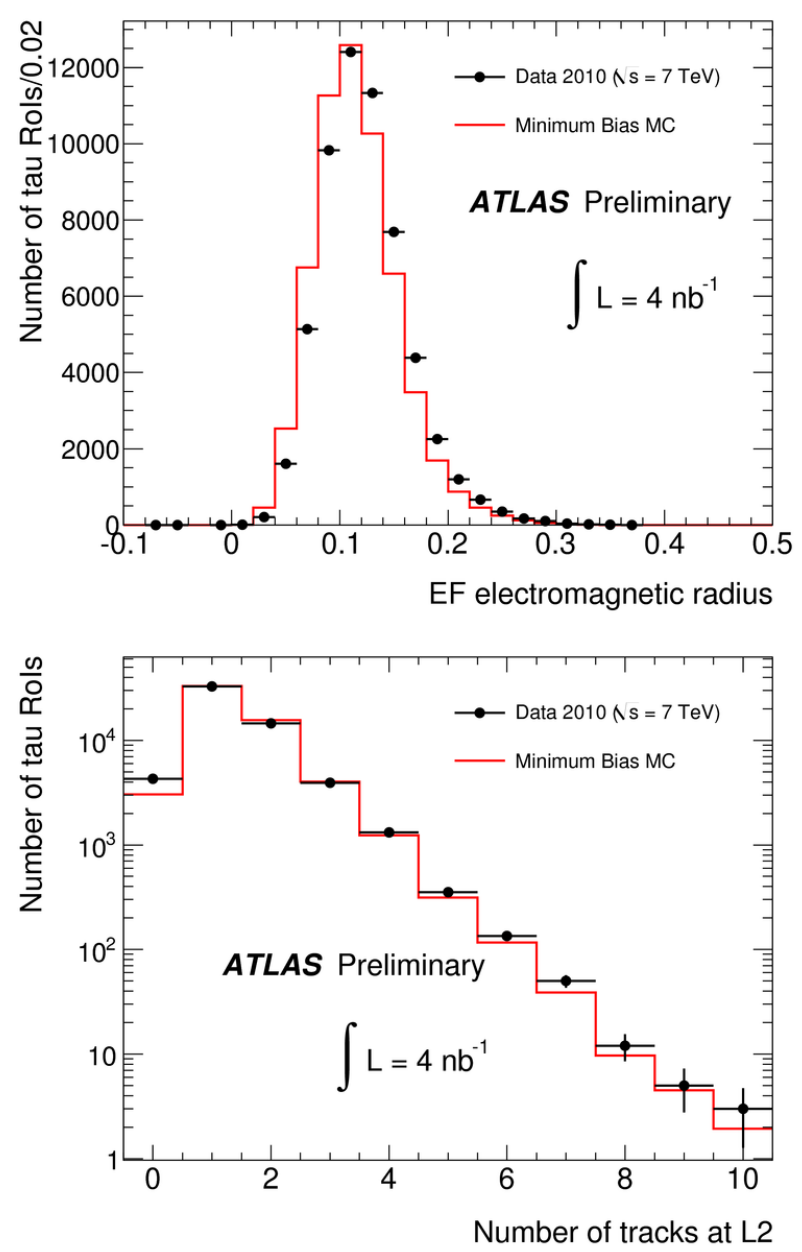

Figure 8. Comparison of the Monte-Carlo and the $\sqrt{s}=7 \mathrm{TeV}$ collisions data for two variables used by the HLT to identify hadronically decaying tau leptons. On the top is the EM radius, which is a measure of the size of the tau-jet, for the Event Filter level. On the bottom is the track multiplicity at Level 2.

The EF looks for the same properties using refined algorithms, similar to the offline reconstruction ones, to build tracks and topological clusters on a window around the RoI position, and selects taus with different criteria for the 1prong and the multi-prong decays. In Fig.8 comparisons for discriminating variables for the HLT are shown, between the data and Monte-Carlo simulation, showing good agreement overall. 


\section{CONCLUSIONS}

The ATLAS trigger selection for the electron, photon and tau has shown good performance during the 2010 data-taking period. The L1 selections show good efficiency and agree very well with simulations. The system has been running in stable mode, with the HLT fully active, since July 2010. These triggers have provided good event selection for ongoing analyses by the collaboration.

\section{REFERENCES}

[1] The ATLAS Collaboration, G. Aad et al., The ATLAS Experiment at the CERN Large Hadron Collider, JINST 3, S08003 (2008).

[2] The ATLAS Collaboration, G. Aad et al., Charged-particle multiplicities in pp interactions at sqrt $(s)=900 \mathrm{GeV}$ measured with the ATLAS detector at the LHC, Phys. Lett. B 688, 21 (2010) [arXiv:1003.3124 [hep-ex]].

[3] The ATLAS Collaboration, W. Fedorko, Triggering on $7 \mathrm{TeV}$ Collisions with the ATLAS High Level Trigger, these proceedings.

[4] The ATLAS Collaboration, A. Oh, Commissioning of the ATLAS Muon Trigger with Beam Collisions at the LHC, these proceedings.

[5] The ATLAS Collaboration, G. Aad et al., Performance of the ATLAS tau trigger in $p$ - $p$ collisions at $\sqrt{s}=7 \mathrm{TeV}$, ATLAS-CONF-2010-090, Geneva (2010).

[6] The ATLAS Collaboration, G. Aad et al., Performance of the Electron and Photon Trigger in p-p Collisions at $\sqrt{s}=900 \mathrm{GeV}$, ATLAS-CONF-2010-022, Geneva (2010).

[7] The ATLAS Collaboration, G. Aad et al., Expected performance of the ATLAS experiment: detector, trigger and physics, CERN-OPEN-2008-020, arXiv: 0901.0512 [hep-ex] (2008). 